\title{
Adhesive bonding of measurement equipment on impact-driven offshore monopile foundations
}

\author{
Gregor Wisner ${ }^{1 *}$, Elisabeth Stammen ${ }^{1}$, Klaus Dilger $^{1}$, Hauke Sychla², Philipp Stein², Christian Kornemann ${ }^{2}$ \\ and Jörg Gattermann²
}

*Correspondence:

g.wisner@tu-braunschweig.de

${ }^{1}$ Institute of Joining

and Welding of Technische

Universität Braunschweig

IFS, Langer Kamp 8,

38106 Braunschweig, Germany

Full list of author information

is available at the end of the article

\begin{abstract}
To a certain extent, adhesive bonding of measurement equipment is very common in science and technology, e.g. adhesive bonding of small-scale strain gauges. Adhesive bonding of the entire equipment for a fully autonomous pile driving monitoring of an impact-driven large-scale foundation structure for an offshore wind farm is a completely new application method. Several offshore wind farms are currently under construction in the North and Baltic Seas. Impact pile driving of the large-scale foundations usually causes much louder noise than permitted by regulations, so methods for noise reduction are necessary. Geotechnical engineers of the TU Braunschweig are investigating combined methods for reducing that noise, and in 2014 they had the opportunity to install measurement equipment for the investigation of dynamic pile deflections during pile driving into three of in total eighty monopiles (length: $60 \mathrm{~m}$, diameter: $6 \mathrm{~m}$ ) of an offshore wind farm in the German North Sea. Due to certification issues conventional methods of fastening such as screwing or welding were not permitted. Instead, adhesive bonding of all parts (sensors, cables, shielding, recorder/computer) was successfully applied and withstood impact driving with several thousand blows of up to $1200 \mathrm{~g}$ (earth gravity). The authors would like to present the concept and preceding tests of the adhesive bonding applied within the research project 'triad'.
\end{abstract}

Keywords: Impact-driven pile, Pile driving monitoring, PDM, Dynamic pile test, DPT, Noise emission, Adhesive bonding, Elastic bonding, Sensor application, Thick film adhesive, PUR, Boosted PUR, Primer, Impact test, Repetitive impact testing

\section{Motivation}

The instrumentation with measurement equipment of impact-driven piles is often necessary in industrial and scientific projects. One application is pile driving monitoring during the installation of piles or dynamic pile tests after a certain time after installation to estimate the bearing capacity of a pile. Prefabricated piles (mostly steel profiles) are driven into the soil by using impact hammers. A pile driving monitoring can be carried out during installation. Concrete piles that are cast in place are tested by dynamic pile tests after the concrete has hardened. The results are often used to give proof of the stability of foundations for buildings. The techniques require the application of strain gauges and accelerometers to the pile which are commonly used by drilling holes into

(c) 2015 Wisner et al. This article is distributed under the terms of the Creative Commons Attribution 4.0 International License (http:// creativecommons.org/licenses/by/4.0/), which permits unrestricted use, distribution, and reproduction in any medium, provided you give appropriate credit to the original author(s) and the source, provide a link to the Creative Commons license, and indicate if changes were made. 
the piles and fastening the sensors by screws. With steel piles, welding of additional plates is also possible to avoid drilling into structural steel.

From the characteristics of strain waves and velocities travelling through the pile, the bearing capacity of the pile can be estimated. Figure 1 shows the force velocity diagram of an impact-driven steel pile. The red and green lines indicate the points in time when the elastic wave in the pile reaches the sensor plane for the first time after impact and the second time after being reflected at the pile toe, respectively.

The force (black line) is calculated from strain measurements multiplied by the piles cross section and Young's modulus (Eq. 1), while the product of velocity $\times$ impedance (blue line) is calculated from integrated acceleration measurements and the cross sectional and material properties of the pile (see Eq. 2):

$$
\begin{aligned}
& F=\varepsilon \times E \times A \quad[k N] \\
& v \times z=\int a d t \times A \sqrt{E \times \rho} \quad[k N]
\end{aligned}
$$

with: F, force $(\mathrm{kN})$; E, Young's modulus of elasticity of pile material $\left(\mathrm{kN} / \mathrm{m}^{2}\right) ; \varepsilon$, strain reading $(-)$; A, cross sectional area of the pile $\left(\mathrm{m}^{2}\right)$; t time $(\mathrm{s})$; v, velocity $(\mathrm{m} / \mathrm{s})$; $\mathrm{z}$, impedance of the pile $(\mathrm{kNs} / \mathrm{m}) ; \rho$, unit weight of the pile material $\left(\mathrm{kg} / \mathrm{m}^{3}\right)$.

Both, the force from strain measurements and velocity $\times$ impedance have the dimension of a force $(\mathrm{N}$ or $\mathrm{kN})$. The static-bearing capacity $\mathrm{R}_{\text {stat }}$ of the pile can be calculated with the difference of the total and the dynamic resistance at time steps $t_{1}$ (red line) and $\mathrm{t}_{2}$ (green line):

$$
R_{\text {stat }}=R_{\text {tot }}-R_{d y n} \quad[k N]
$$

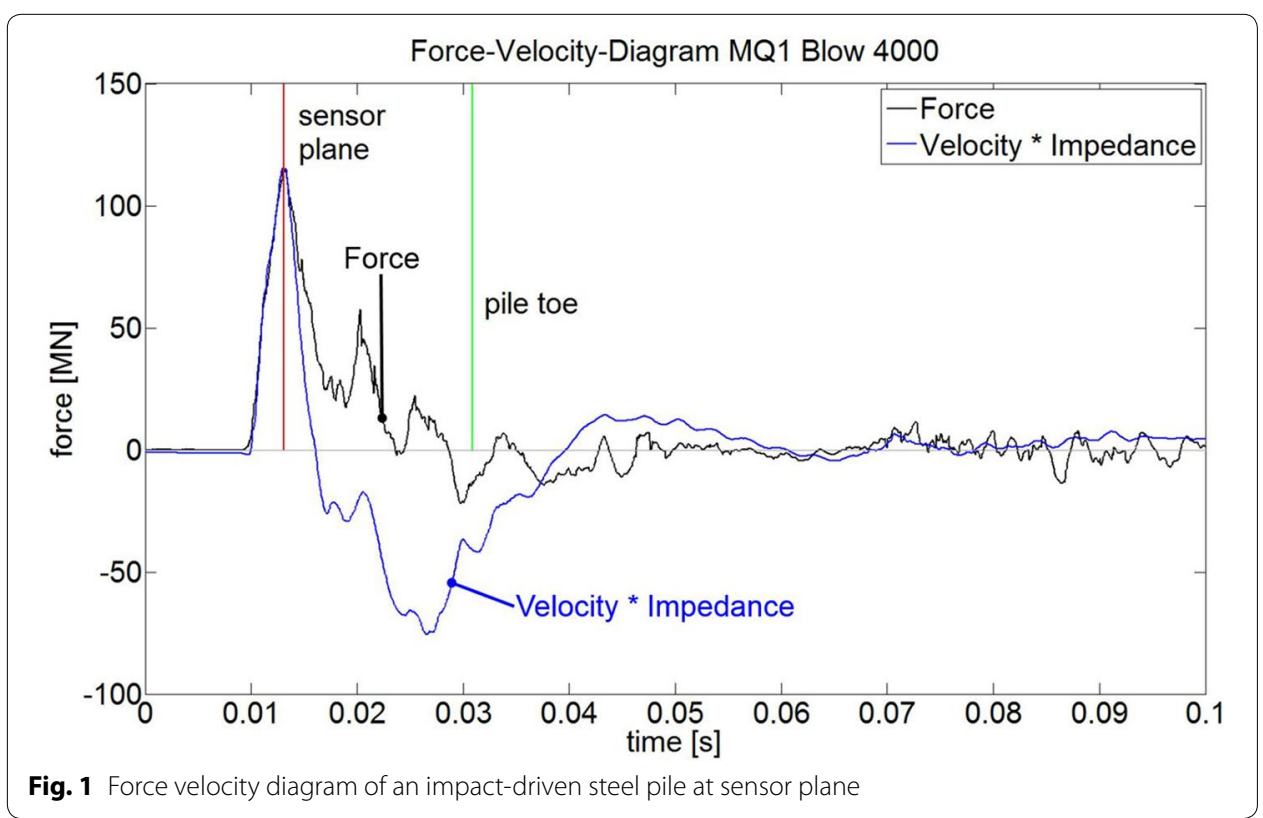


where

$$
R_{t o t}=\frac{1}{2}\left(F_{1}+Z \times v_{1}\right)+\frac{1}{2}\left(F_{2}-Z \times v_{2}\right) \quad[k N]
$$

and

$$
R_{d y n}=J_{c} \times Z \times v_{b} \quad[k N]
$$

with: $J_{c}$, damping factor $(-), v_{b}$, penetration velocity at pile toe $(\mathrm{m} / \mathrm{s})$. A more sophisticated approach to determine a pile's bearing capacity is obtained by fitting a numerical one-dimensional model to the measurements in recursive iteration. Details on the theory of pile dynamics and advanced modelling can be found in [1].

In current research on underwater noise emissions during the installation of pile foundations for offshore wind turbines, the wave propagation in the entire system of pile, soil and seawater is investigated. Geotechnical engineers of the TU Braunschweig are investigating combined methods for reducing that noise during the installation of an offshore wind farm in the German North Sea [2]. Figure 2 shows a schematic drawing of the different modes of wave propagation during offshore pile driving and details of the instrumentation of the pile.

Therefore, strain gauges and accelerometers (acc) as well as an autarkic data acquisition unit had to be installed inside large monopiles of a recently built off-shore wind farm in the North Sea. Those monopiles are open-ended steel tube piles with diameters of about $6 \mathrm{~m}$ and lengths of 55-60 m, depending on the position in the wind farm. Since the different measurement sections (MS) where distributed along the length of the pile,

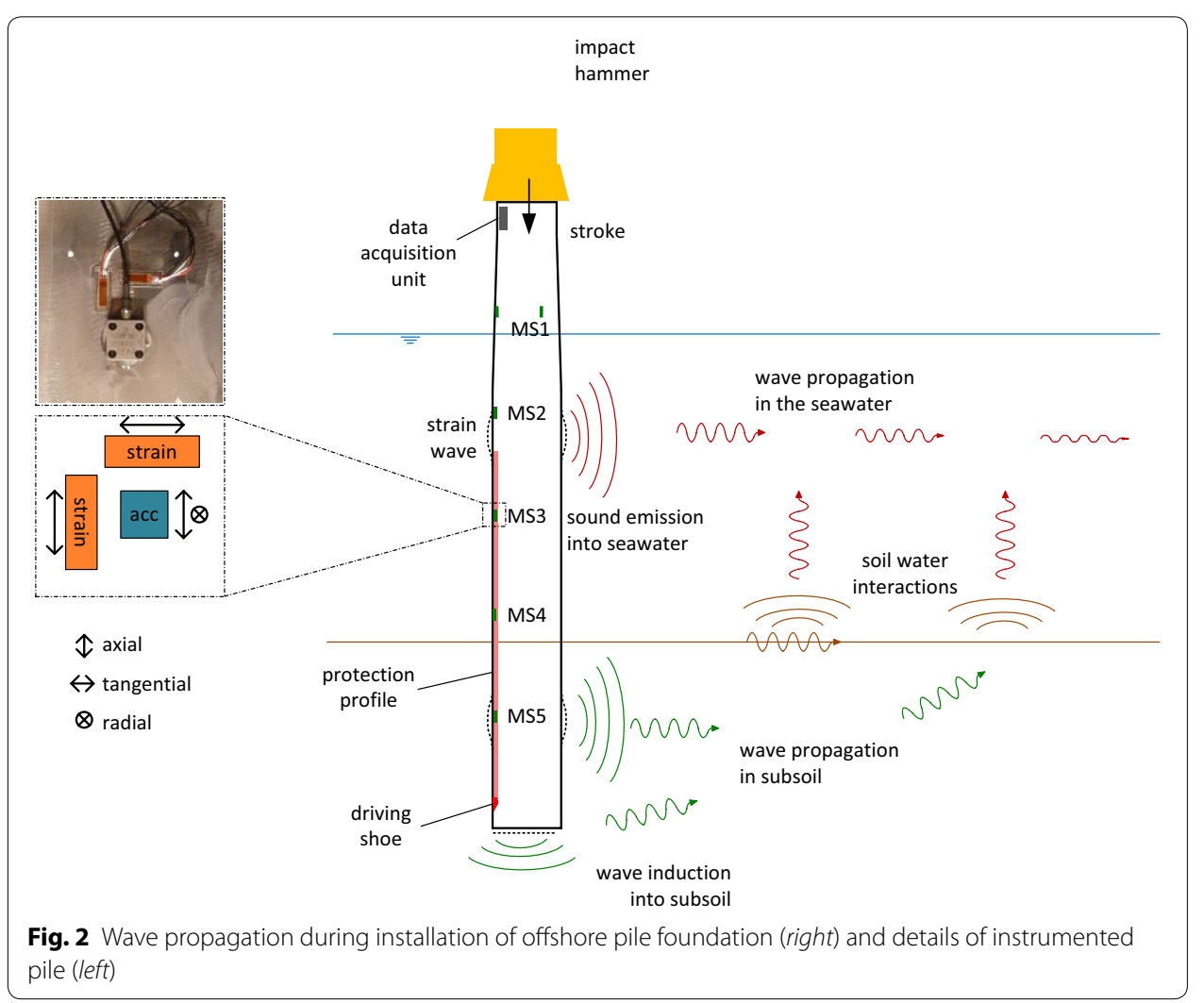


a mechanical protection of the measurement equipment against the penetration into the soil had to be installed, too.

Due to the high certification standards of offshore constructions and the already completed design phase of the monopiles, neither welding nor drilling into the piles was possible. This was not an issue for the installation of the sensors themselves since the application of miniature strain gauges and accelerometers by means of thin layer adhesives or spot welding is common practice. However, the fastening of a $40 \mathrm{~kg}$ data acquisition unit, several hundreds of meters of cable and steel profiles for the protection of sensors and cables to a pile wall that would be driven with energies of more than $1000 \mathrm{~kJ}$ per blow and accelerations of more than $1000 \mathrm{~g}$ (earth gravity) was a big challenge. In close cooperation, the Institute of Joining and Welding and the Institute for Soil Mechanics and Foundation Engineering of Technische Universität Braunschweig in Germany developed a method by adhesive bonding of all required components for a scientific pile driving monitoring, which was successfully used during the installation of three large-scale monopiles.

\section{Adhesive technology}

\section{General technical approach}

After the basic decision of bonding all components to the monopiles, two main adhesive routes were implemented for the small sensors on the one hand and all other components like cables, protection profiles and recording computer on the other hand. Small sensors were applied by means of thin structural layers of adhesives for best coupling to the structure. This method is state of the art for measuring strains and accelerations in laboratory scale and this is also easily adaptable to larger scales. Thin layers and rigid bonds are necessary to avoid mechanical damping of the layer between sensors and structure, to guarantee correct measurements of dynamic deformation of the structure. All other components including sensor protection, cables, monitoring equipment, etc. were installed using thick layers of semi-structural adhesive on a maximized area to provide elastic bedding with excellent adhesion, high damping factor and low failure growth.

Those two routes of adhesive bonding obviously require completely different materials. Because of the well-established adhesive bonding of small sized sensors such as strain gauges or other sensors the research for the best material combination was carried out by using the recommended adhesives along with recommended primers for the grinded pile surface of structural grade carbon steel (S355ML/NL acc. EN 10025-4:2004) and testing it for sufficient results. The small sensors have very low mass and a comparatively large contact surface to bond, e.g. strain gauges as thin films.

The much greater challenge lay in any other part with larger dimensions and a much higher mass than the small sized sensors. Any mass was going to be accelerated by every blow of the pile driving process with a high number of repetitions. Initial information from previous investigations on pile driving of much smaller structures were reported to be in the region of up to $800 \mathrm{~g}$ (earth gravity) of acceleration. One solution to solve this problem is giving up the rigidity of the bond and giving way to several extra degrees of freedom and also profiting from the damping factor of soft semi-structural materials to ease the high-energy impacts on the adhesively bonded parts. The main strategy was to 
bond all heavy masses on a maximal surface area with a thick layer of soft semi-structural adhesive and also break down large structures into smaller pieces to avoid flexural interactions while moving under the accelerations and carry out free movements on the relatively thick polymer beddings. For an easy field application, a one-part moisturecured polyurethane adhesive with a booster component, applied with a simple double cartridge (mixing ratio of 1:10) and static mixer was favoured. Laboratory tests were carried out to check the suitability of the chosen products on all related surfaces by using appropriate primer materials.

To find out whether an adhesive material is suitable for the thick layer bonding, two different kinds of testing were carried out on the most important substrates. The most common method of testing an adhesive joint is a single overlapped specimen tested in a quasi-static way to achieve an ultimate load and to watch the fracture pattern. Due to the fact that all bonded equipment should withstand high impacts being applied on the monopile wall, an appropriate test for the assessment of resistance against high impact energy on small specimens should complement the preliminary testing. One of the components with the largest single mass of the equipment to be bonded on the monopole wall was judged as most critical for adhesive bonding. Therefore a test campaign to estimate the feasibility of successful bonding application was planned. A similar energy impact on an adhesively bonded mass of a mounting plate $\left(1 \mathrm{~m}^{2}\right)$ and attached computer box as data acquisition unit (total mass $66 \mathrm{~kg}$ ) was calculated for the analogue approach with a small Charpy testing machine and two different hammers to operate with. The result was an impact campaign of a certain number of repeatable blows and each blow caused a maximum dynamic shear stress of a limited stress of about $5 \mathrm{MPa}$. The calculative approach of the analogue energy impact is described as follows.

\section{Theoretical calculation of initiated impact energy for an analogue approach to test small-scale impact specimens}

For the experiments in small-scale tests the scaled input values of the pile driving energy and the pile cross-section area were of prime importance. So the calculation of an equivalent impact energy was the first step for the small-scale tests. In this case the penetration record of previously driven monopoles of the wind energy farm and the associated blueprint were used as design criteria for calculation. The derivation of the real terms and conditions during the pile driving into a small-scaled test should be clearly represented in the following part. The relevant parts of the penetration record of the driven monopile and the associated blueprint are shown in the following Table 1.

In the preliminary tests it was the thought to use a Charpy machine and a swing hammer with a mass of $1.983 \mathrm{~kg}$ (subsequently designated as "small swing hammer"). The first experiments showed that the mass of the small swing hammer did not suffice. Because of this fact another swing hammer with a mass of $6.610 \mathrm{~kg}$ was implemented in the small-scale tests (subsequently designated as "large swing hammer"). The height of fall for both swing hammers was $0.756 \mathrm{~m}$.

In a few tests the mean force of both swing hammers was calculated at 3000 and $8000 \mathrm{~N}$. The amount of the impact energy in relation to the impact energy calculated (in Table 1) and the area of adherent are shown in the following Table 2. 
Table 1 Calculation of the mean driving energy

\begin{tabular}{lll}
\hline Mean driving energy & & \\
\hline Sum of driving energy & 78,911 & $\mathrm{~kJ}$ \\
Total number of blows & 4245 & $\mathrm{~cm}$ \\
Outside diameter (monopile) & 522 & $\mathrm{~cm}$ \\
Inside diameter (monopile) & 507 & $\mathrm{~cm}$ \\
Diameter (steel profile) & 7.5 & $\mathrm{~cm}^{2}$ \\
Resultant cross-sectional area & $12,122.62$ & $\mathrm{~J} / \mathrm{cm}^{2}$ \\
Impact energy & 6509.40 & $\mathrm{~J} / \mathrm{cm}^{2}$ \\
Mean impact energy per blow & 1.53 &
\end{tabular}

Table 2 Calculation of the impact energy

\begin{tabular}{lllll}
\hline Mean force per pendulum blow & \multicolumn{2}{l}{ Small hammer } & & \multicolumn{2}{l}{ Large hammer } \\
\cline { 2 - 5 } & $\mathbf{3 0 0 0}$ & $\mathbf{N}$ & $\mathbf{8 0 0 0}$ & $\mathbf{N}$ \\
\hline Height of fall & 0.75648 & $\mathrm{~m}$ & 0.75648 & $\mathrm{~m}$ \\
Area of adherent & 36 & $\mathrm{~cm}^{2}$ & 36 & $\mathrm{~cm}^{2}$ \\
Resulting energy & 2269.44 & $\mathrm{~J}(\mathrm{Nm})$ & 6051.84 & $\mathrm{~J}(\mathrm{Nm})$ \\
Impact energy & 63.04 & $\mathrm{~J} / \mathrm{cm}^{2}$ & 168.11 & $\mathrm{~J} / \mathrm{cm}^{2}$ \\
\hline
\end{tabular}

With the parameters calculated in Tables 1 and 2 a new factor was determined, the blow factor, for the calculation of the substitutional number of blows in the next step. This new parameter is made up of the quotient of impact energy and the mean impact energy per blow. With the number of blows from the penetration record of the driven full scale monopile and the calculated factor it is possible to determine the substitutional number of blows. The calculation is shown in the following Table 3.

The calculation of the substitutional number of blows shows that 104 blows would be necessary with the small swing hammer and 39 blows with the large one to reproduce the original number of blows during the pile driving. At this point, it should be mentioned that the calculation of the substitutional number of blows is only a theoretical value. There are a lot of other factors during the pile drive process and the small-scale tests which could not be considered.

\section{Preliminary testing}

\section{Impact testing}

Studies on a simple Charpy impact test machine were carried out to estimate the adhesive behaviour under very high dynamic loading. Based on experiences of the blow count (up to 5000 blows) and impact energy (up to $2000 \mathrm{~kJ}$ ) of monopile installations on the

Table 3 Substitutional number of blows for the impact testing machine

\begin{tabular}{lll}
\hline & Small hammer & Large hammer \\
\hline Blow factor & 41.11 & 109.63 \\
Substitutional number of blows & 103.26 & 38.72 \\
Chosen blows & 104 & 39 \\
\hline
\end{tabular}


one hand and the bonding areas of the real structures and specimens on the other, an estimation of specific analogue energy for a very limited number of repetitive impacts was calculated in the former chapter.

The design of a double-sided adhesively bonded specimen for repetitive impact campaigns in a Charpy machine is shown in Fig. 3. The design allows the mounting of different substrates within the $\mathrm{H}$-shaped specimen. The most important substrates were uncoated, sanded steel sheet and steel sheet with an epoxy coating. These substrates were provided as thick sheet metal of about $5 \mathrm{~mm}$. The total bonding area was $3600 \mathrm{~mm}^{2}$ (both sides) and adhesive layer thicknesses of up to $10 \mathrm{~mm}$ each were possible (with very thin substrates). Most specimens were bonded with a $5 \mathrm{~mm}$ adhesive layer thickness on 5 mm substrates.

Specimens were bonded on a specific assembly station, where the two pre-assembled angles with the substrate (uncoated or epoxy-coated steel sheet, structurally bonded with a 2-part epoxy adhesive) and the $\mathrm{H}$-shaped centre piece were fixed for a reproducible specimen geometry. Adhesive bonding of the moisture-curable PUR on pre-coated primers was carried out according to the manufacturers' recommendations. The boosted adhesive system with an open time of up to $30 \mathrm{~min}$ (maximum for field applications)

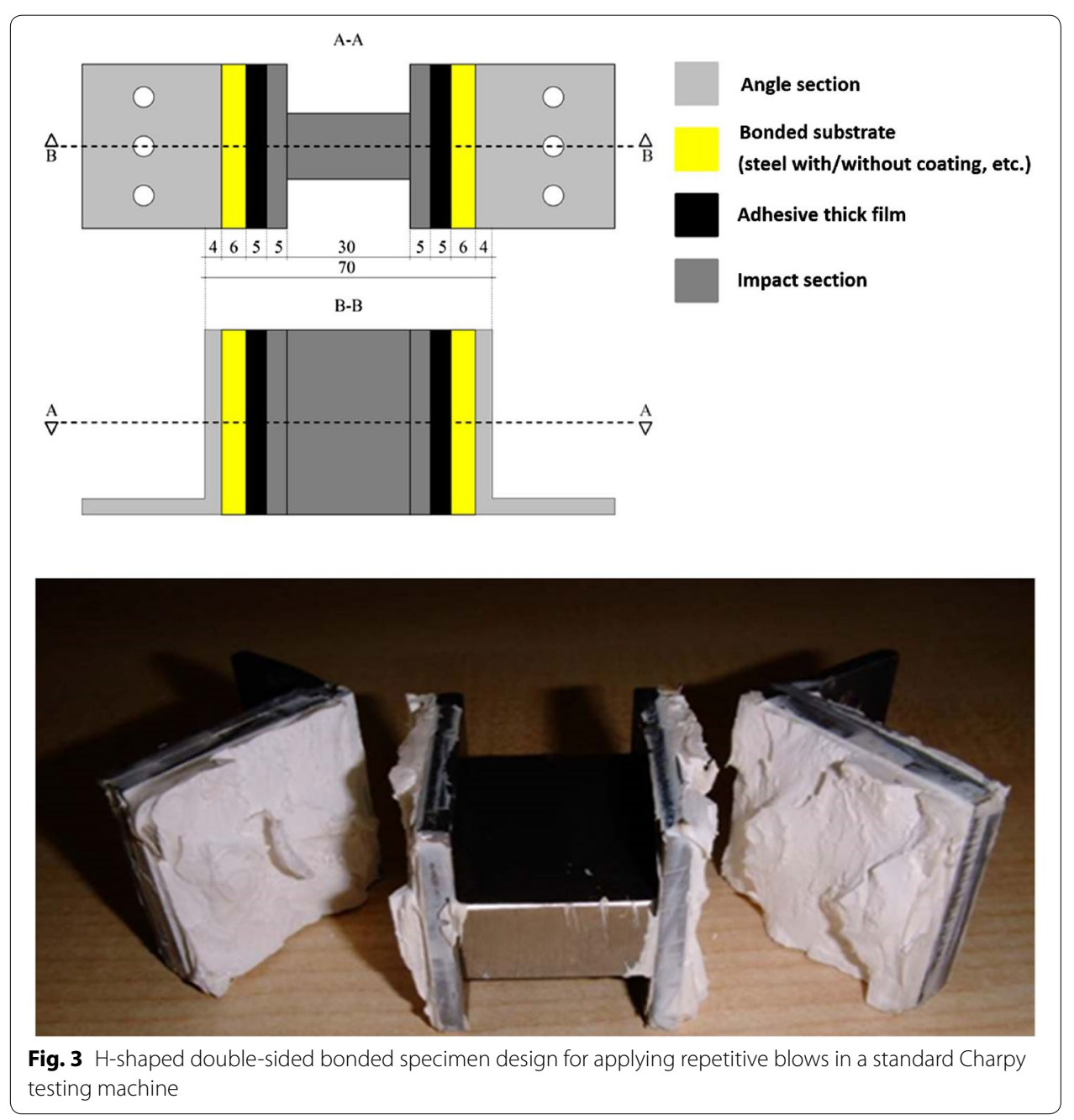


tends to cure relatively fast, due to the moisture content in the booster paste. However, bonded specimens were cured for at least 2 days before a repetitive impact campaign.

A small Charpy machine with a maximal space for mounting a versatile specimen design was available for the tests, see Fig. 4. Two hammers with different masses ( 2 and $6.6 \mathrm{~kg}$ ) were equipped with strain gauges close to the impact area. Based on this equipment, an H-shaped specimen design for being installed directly into the Charpy machine by using existing fastening items was sketched. The Charpy testing machine is recommended for impact testing in adhesive technology in addition to the originally intended testing method to provide material data for metals and plastics by direct destructive testing [3]. The Wedge impact peel test acc. ISO 11343 [4] is well known in different industries using adhesive bonding of thin sheets and interested in high strain behaviour (crash) of the bonds. Adams [5] also recommended the Charpy machine for applying high strain on the more common single overlapped shear test. Due to the fact that the specimens should not be destroyed by the one and only impact, a compact design of a specimen with a large bonding area using two symmetrically positioned adhesive layers was considered to be of more practical use in the Charpy machine.

Applying defined repetitive non-destructive impacts on a Charpy machine was only possible by assuring just single impacts from full height and stopping the hammer on the high point of counter reversing. Due to the small size of the Charpy machine, the task of catching the reversing hammer, after the first impact at the high point of movement, was done manually by the user. If a failure of the thick elastic layer occurred after a number of blows, this was observed during the specific campaign, but a visible crack was not immediately reported by the signals of the strain gauges of the hammer. Only after a few extra blows, a modified signal in the recorded measurements was detected afterwards. This was explained by the limited sensitivity of the method. Figure 5 shows an example of a specimen with a very short life of only 18 blows until total failure.

The signal in general consists in the first sharp and high impact of the hammer as first contact and immediately stopping the hammer by transferring the energy into the specimen. The specimen deforms and the adhesive layers are strained by shear. The reverse

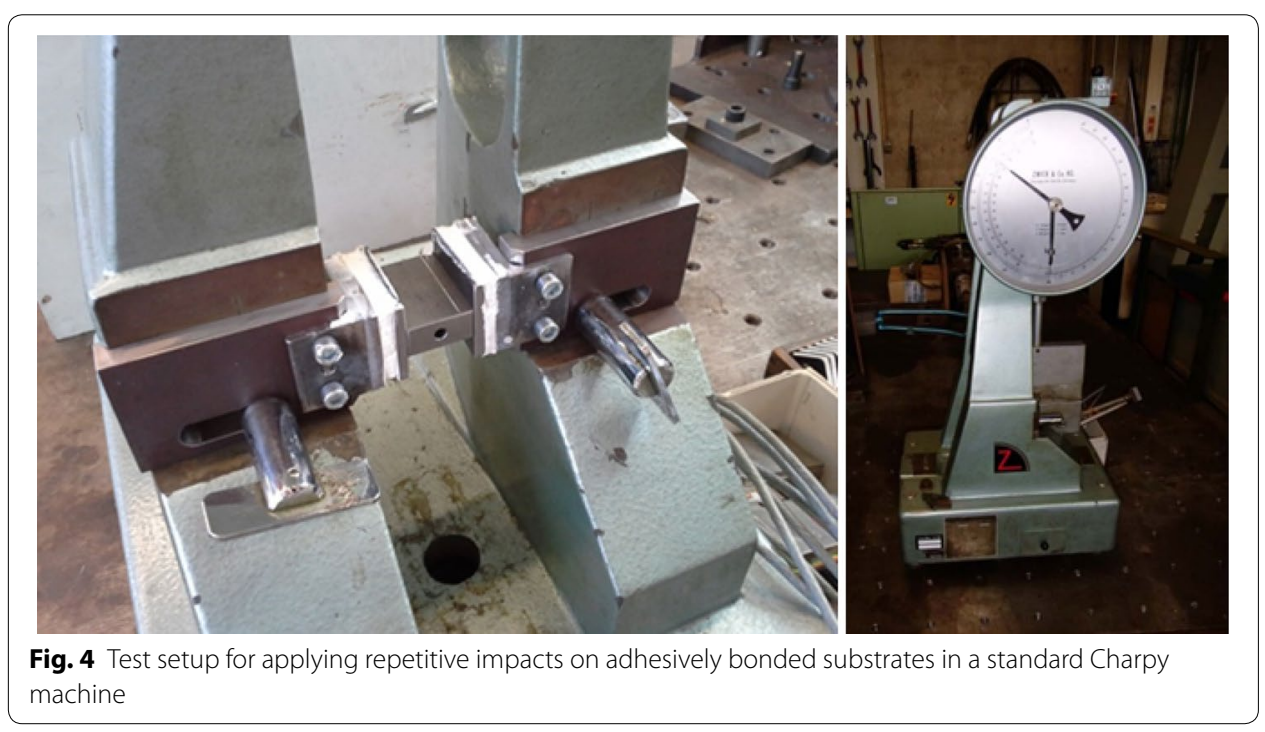




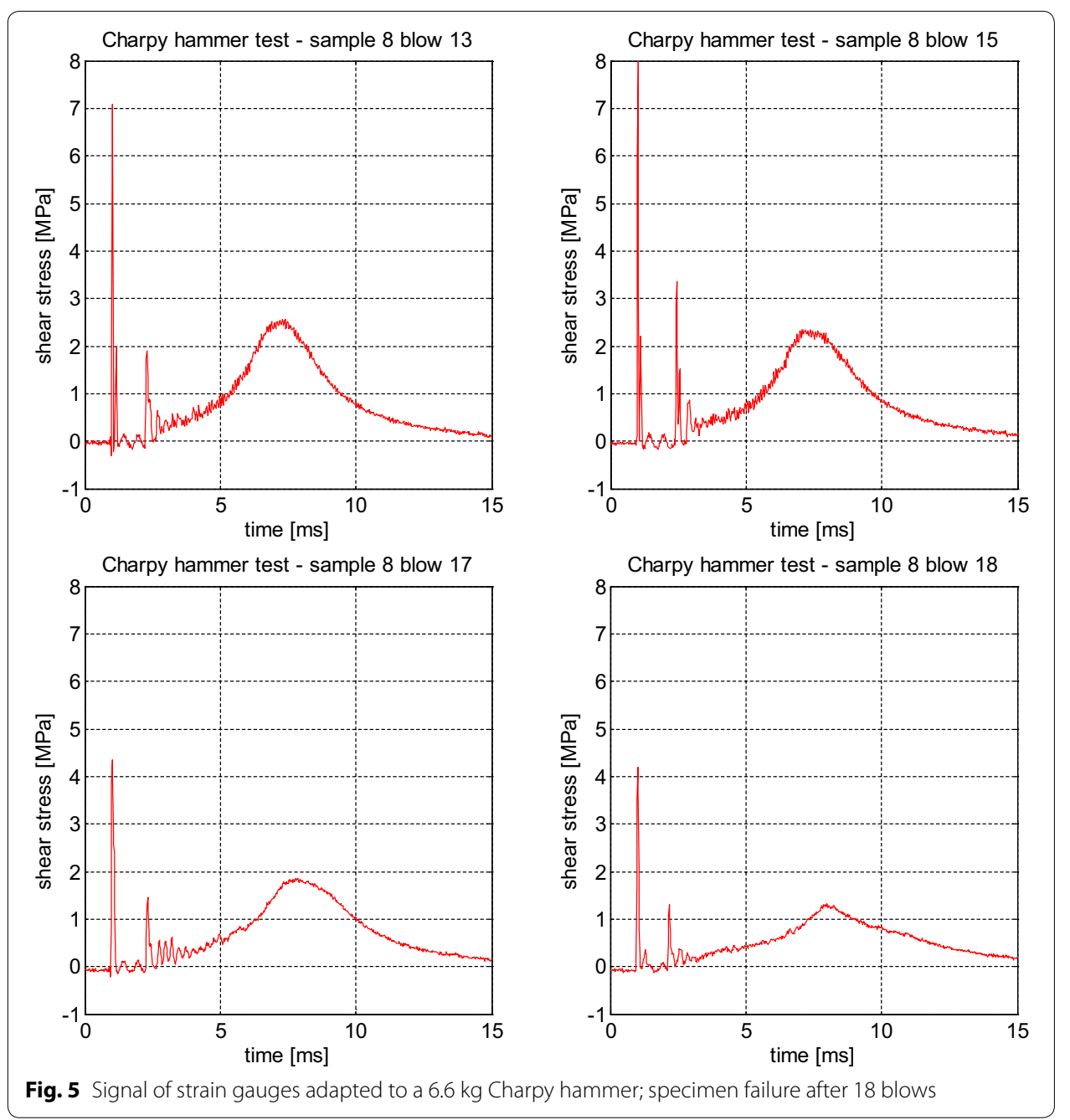

movement of the $\mathrm{H}$-shaped centre piece hits the hammer only after a millisecond and starts accelerating the hammer in the reverse direction. The signal also shows a certain bouncing between specimen and hammer at this accelerating movement. This can be shown exemplary with the signal sequence of $\mathrm{H}$-shaped specimen number 8 . The specimen was repeatedly impacted by the $6.6 \mathrm{~kg}$ hammer and after blow no. 13, a small crack was observed on the left adhesive layer. The signal was the same as with any other blow recorded before (see Fig. 5, top left). With the following blows, another crack was observed in the right adhesive layer, the signal was slightly modified by broadening the last peak (blows 15 and 17, see Fig. 5, top right and bottom left). After blow no. 18 (see, Fig. 5, bottom right), the $\mathrm{H}$-shaped centre piece was fully debonded.

During the impact campaign on $\mathrm{H}$-shaped specimens a major problem with the auxiliary bonding of the substrates to the mounting angles occurred in a number of specimens. Due to the fact of most versatile design a structural bonding of the particular substrate to the mounting angles was chosen. Structural bonding is best by applying thin adhesive films with a significant strength and rigidity, like adhesives based on epoxy, acrylics or phenolic resins. Different epoxy based adhesives were tried out and surface 
modifications like grinding and degreasing were applied to react on the interim results of the campaign. It turned out, that a number of specimens failed early in the auxiliary bonding and could not be tested further. After close examination of the thick film adhesive layers for visible cracks, a repair bonding on the auxiliary joint was established and the particular specimen (marked as a repaired one) could perform another test run. In some cases, a mixed failure in both types of bondlines occurred after a certain number of blows. Those specimens could not be repaired because of cracks in the thick adhesive layer. Table 4 gives an overview on this campaign.

In the end, a fully optimized auxiliary bonding was not established successfully within the test campaign preliminary to the major application campaign on the construction site. This result emphasises, that an adhesive bonding with conventional rigid and high strength adhesives is not suitable for larger masses, when applied with very high impact energy and huge accelerations. This kind of adhesive bonding is only suitable for very small masses such as the sensors itself. Therefore the fastening of substrates in the displayed $\mathrm{H}$-shaped specimen design should be optimized further on.

\section{Quasi-static testing}

Quasi-static tests were carried out to find out the strength, deformation behaviour and the fracture pattern of the used adhesive on pure steel substrate and epoxy coated steel, see Fig. 6. A number of specimens (five per series) were bonded with rubber spacers to provide the defined layer thickness of a $3 \mathrm{~mm}$ series and a $5 \mathrm{~mm}$ series with the same adhesive and two different primers. One (transparent) primer was most suitable for metallic substrates, for all polymer materials a lacquer like black primer was recommended by the manufacturer. A quasi-static shear strength of about $0.8 \mathrm{MPa}$ was measured at a shear deformation between 200 and $300 \%$, see Fig. 6 left. The failure modes were cohesive in all cases within the adhesive which was important to avoid any damage to the coating of the monopiles applied at the upper part of the piles. In the case of failure of the adhesive, the measuring equipment would fall off without damaging parts of the coating.

Table 4 Overview on repetitive impact campaign on grinded steel substrates (H-shaped specimen in Charpy machine)

\begin{tabular}{llrl}
\hline Experiment no & Impact hammer type & Number of blows & $\begin{array}{l}\text { Failure in specific type } \\
\text { of adhesive layer }\end{array}$ \\
\hline 1 & P1 (2 kg hammer) & 54 & No failure \\
2 & P2 (6.6 kg hammer) & 23 & Auxiliary bonding \\
3 & P2 & 2 & Auxiliary bonding \\
$3 a$ (repaired aux. bond) & P2 (2nd run) & 18 & Thick film adhesive layer \\
4 & P2 & 1 & Auxiliary bonding \\
$4 a$ (repaired aux. bond) & P2 (2nd run) & 11 & Thick film adhesive layer \\
5 & P2 & 38 & Thick film adhesive layer \\
6 & P2 & 4 & Auxiliary bonding \\
7 & P2 & 32 & Thick film adhesive layer \\
8 & P2 & 19 & Thick film adhesive layer \\
9 & P2 & 7 & Both types of adhesive layer \\
10 & P2 & 2 & Both types of adhesive layer \\
11 & P2 & 9 & Both types of adhesive layer \\
12 & P2 & 10 & Both types of adhesive layer \\
\hline
\end{tabular}



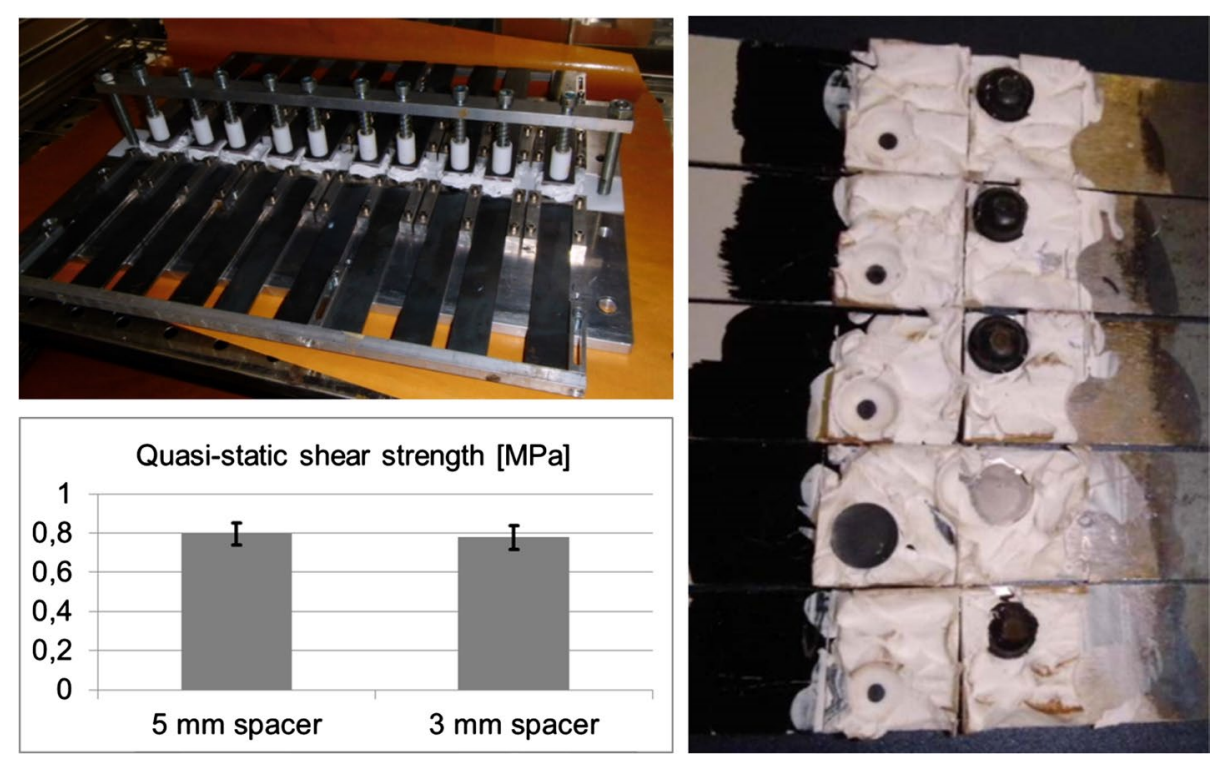

Fig. 6 Preliminary bonding and testing of single overlapped shear specimens testing on quasi-static conditions

Any single overlapped specimen consisted of an epoxy-coated surface plus black-coloured primer on one substrate metal and a sanded and transparent primered surface on the other substrate metal. The relatively large rubber spacers were effectively reducing the bonding surface area on these particular specimens, but those spacers were chosen for assuring a minimal adhesive layer thickness in the real big structures for applying a spacer any 300-500 $\mathrm{mm}$ in a bondline. To apply them with a much greater surface ratio in the small specimens and gaining an average strength of $0.8 \mathrm{MPa}$ was judged as a result on the safe side. The high shear deformation between 200 and $300 \%$ (see Fig. 6 left) is an indicator that dynamic impacts are damped before being transferred from the pile to the applied components. This is of great importance to lessen the impacts of more than $1000 \mathrm{~g}$.

Summarizing the two different testing campaigns on thick film adhesive layers showed different results:

- A small number of specimens reached the theoretical calculated repetitive number of blows with the specific calculated impact energy.

- A number of specimens showed more or less early cracking in the thick adhesive layers and failed after 4-5 additional blows.

- A number of specimens failed early on the structurally bonded thin adhesive layer between the angle piece and the substrate to be tested. This could be improved by changing the adhesive and the process, but was not fully optimized yet.

- Comparing the loads of simple quasi-static single overlapped shear specimens with the impact stresses caused by a single impact by Charpy machine shows a much higher dynamic resistance of the thick elastic layer when applied with high strain loads, see Fig. 7.

- The flexible adhesive layer showed a highly damage-tolerant failure pattern [6, 7]. 


\section{Realising application of measurement equipment}

To provide a suitable substrate for the application of sensors and other components, the steel of the pile was freed from rust over the full length in the measuring axis in a width of about $30 \mathrm{~cm}$. Special attention was paid to the measuring sections where a blank surface was necessary. Both steel and coating were cleaned by alcohol before the application of any components. To achieve best adhesive properties, primers for the different materials (steel, synthetics) were used.

The instrumentation of the first monopile had to take place in winter with low temperatures at the harbour site on the German North Sea. Since the curing of the adhesives needs a minimum temperature of $5{ }^{\circ} \mathrm{C}$ and takes very long time at low temperature, the steel of the pile was heated locally to a moderate temperature and the pile itself was closed up at the ends to provide comfortable working conditions (see Fig. 8).

A commonly used inductive heating system to pre-heat large steel components before welding was installed on the outer surface of the pile. Temperatures of about $20-30{ }^{\circ} \mathrm{C}$ on the inner surface were achieved which led to curing times of about 2-3 days.

After preparation of the pile's surface, strain gauges were applied using a small spot welder and accelerometers were bonded by means of thin adhesive layers. A synthetic cap was then placed over the measuring point and fixed by a thick layer semi-structural adhesive (see Fig. 9, left). (Both cap and substrate had before been treated with primer). The measuring cables were embedded into a thick layer of semi-structural adhesive over the full length. The cables were then covered by a trapezoidal profile at the lower part of the pile where it would penetrate into the soil during piling. The bottom end of the section was closed by a driving shoe (see Fig. 9, right). Cable protection and driving shoe were bonded to the pile by a thick layer semi-structural adhesive as well. The bondline design was established by common recommendations such as given in $[8,9]$ to achieve a maximum strength.

For the realisation of the measurements, an autarkic data acquisition unit with 32 channels and several hours of battery lifetime and storage capacity for high-frequency measurements
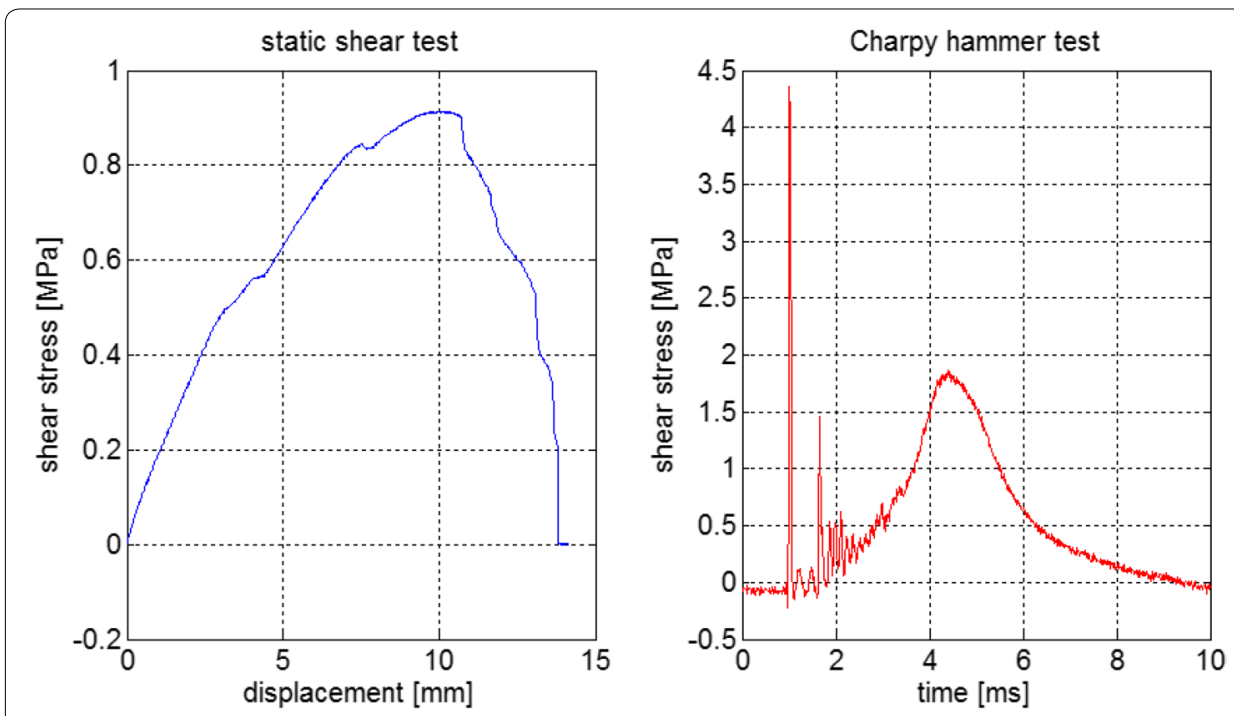

Fig. 7 Characteristic results of laboratory tests on thick layer adhesive: quasi-static test (left) and impact test (right) 

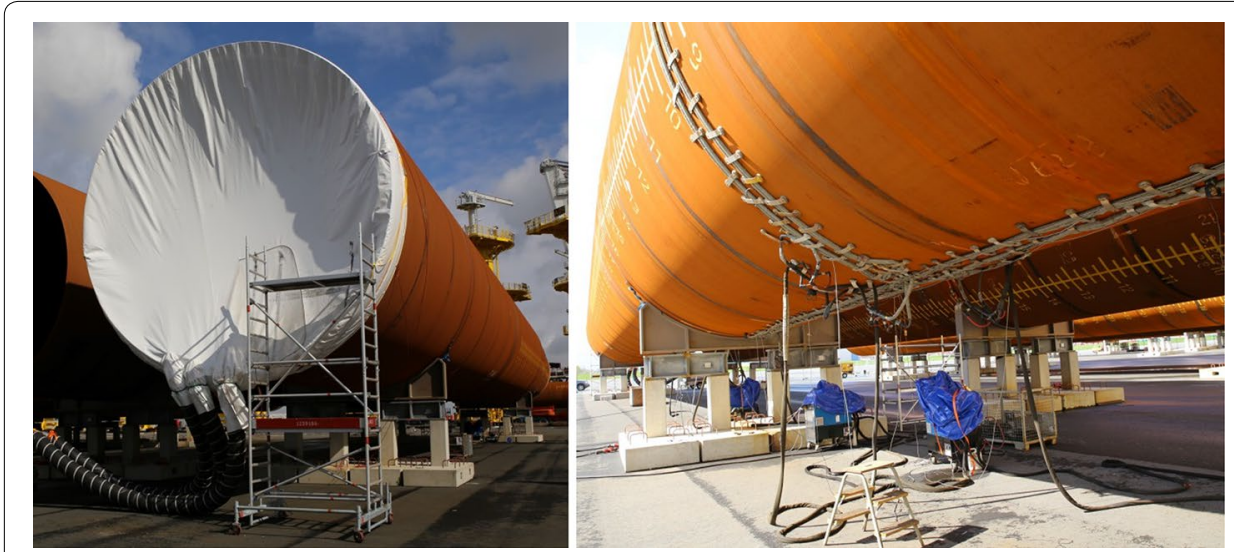

Fig. 8 Closed-up pile with air heating hoses (left) and inductive heating cables alongside the adhesive bonding paths on the inside wall (right)

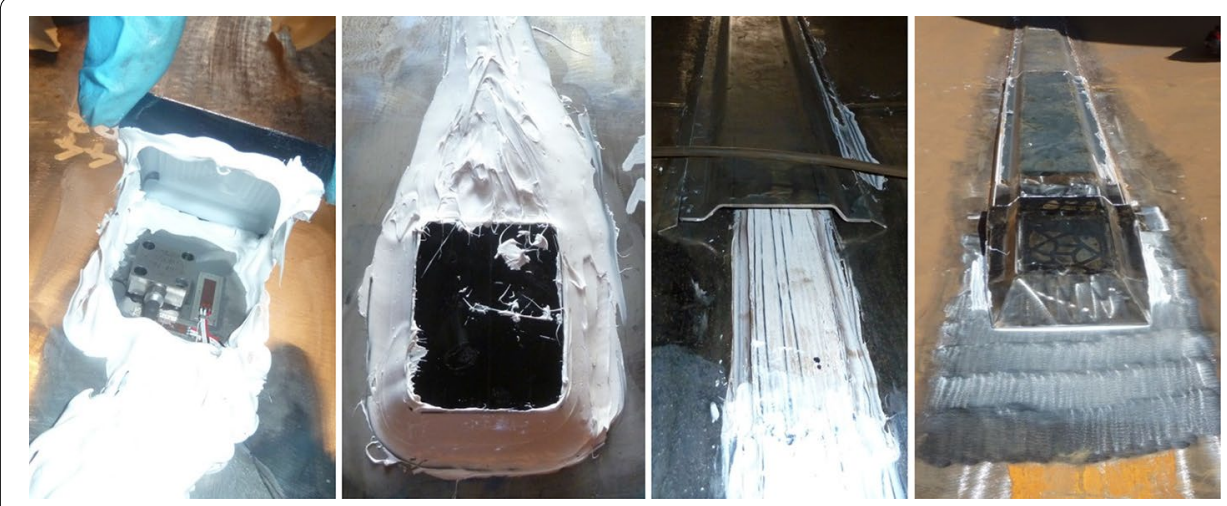

Fig. 9 Sensors below encapsulation, encapsulated sensors, cables embedded in adhesive and cable protection duct, driving shoe at end of cable protection duct near pile toe (from left to right)

had to be designed. The data acquisition had to be installed inside the pile below the pile head to be recovered after the end of pile driving. The data acquisition unit consists of a watertight box containing a measuring computer and battery cells with a total weight of about $40 \mathrm{~kg}$ (see Fig. 10, right). The box was suspended between rubber bands that were connected to a mounting plate. Rubber bands of different stiffness above and below the box created a nonlinear mass-spring system to avoid resonant effects during pile driving.

Since there was no allowance to either screw or weld any lugs to the pile, the mounting plate was bonded by means of a thick layer semi-structural adhesive as well (see Fig. 10, left). The upper part of the monopiles was coated by a corrosion protection which had to stay intact. Thus, no grinding was performed here but the coating was cleaned and the mounting plate as well as all cables and a strain relief were bonded directly to the coating. As stated before, the data acquisition unit and the mounting plate had a weight of about $65 \mathrm{~kg}$.

\section{Results of measuring campaigns during pile driving}

In three measuring campaigns three piles were instrumented by the adhesive method described above. All campaigns were successful with the data acquisition unit logging 


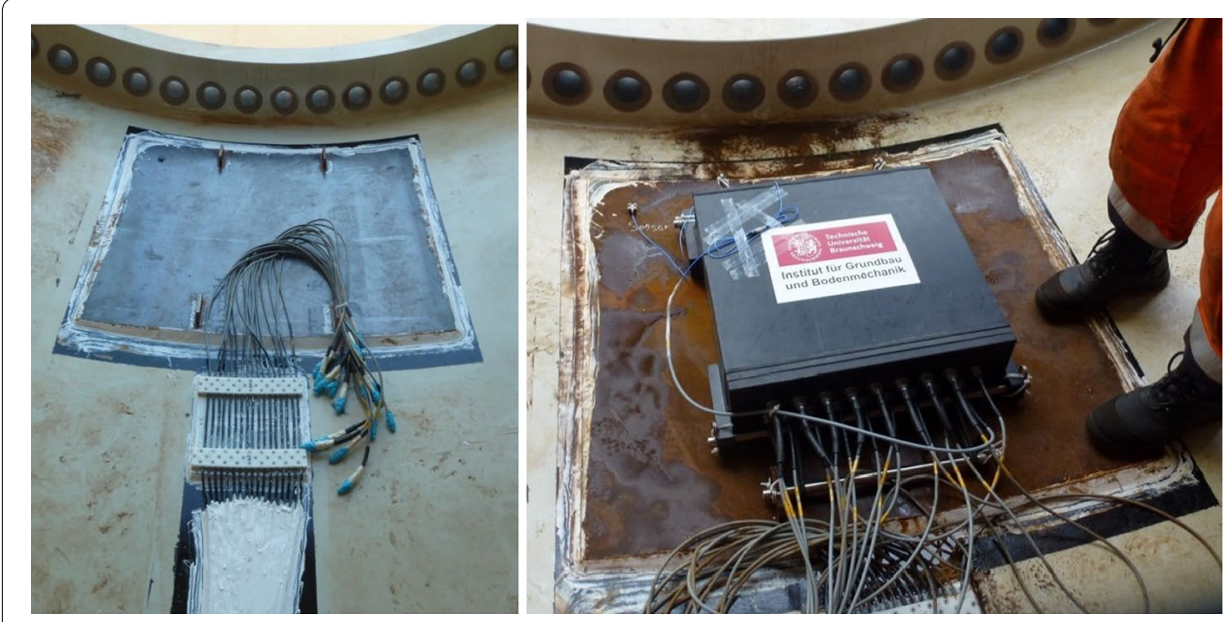

Fig. 10 Adhesively bonded mounting plate for data acquisition unit (left) and mounted acquisition unit before pile driving (right)

data from the first blow to the end of the pile driving process. Plausible data was generated by the majority of the sensors. Visual inspection of the bonding of the mounting plate during recovery of the data acquisition box showed no major damage to the adhesive. However, detailed investigations of any adhesive connections after pile driving were not possible due to limited access or penetration of the sensors into the soil, respectively. In total, the majority of the sensors were fully operational during the whole pile driving process what can be seen in the Fig. 11, which shows the performance of all sensors plotted over penetration.

It can be seen that a minor part of the sensors was not fully operational from the beginning of the measurements, which is most likely due to mistakes made during the assembly of the sensors to the measuring cables or to the data acquisition unit. Also mistakes during the programming of the data acquisition software could be accountable for this. Overall, only a few sensors at only one pile failed during the pile driving. The number of losses during driving is not significantly higher compared to state of the art fastening techniques like screwing or welding.

In one pile, a tri-axial accelerometer was installed to the mounting plate of the data acquisition unit apart from the other sensors in the different sensor planes. Figure 12 shows axial accelerations of the mounting plate ( $\sim 1 \mathrm{~m}$ below pile head; left) and at the first measuring section ( $~ 9 \mathrm{~m}$ below pile head; right) for the same blow towards the end of the pile driving in the time (top) and frequency domain (bottom).

High damping effects regarding acceleration amplitudes due to impact driving by the thick layer adhesive can be seen, especially at higher frequencies. Amplitudes are damped by the factor of approximately 0.5 in general. On the plate, the main vibrations occur in the frequency range below $1000 \mathrm{~Hz}$ while the pile itself vibrates in a wide frequency range between 1000 and $5000 \mathrm{~Hz}$.

\section{Summary}

Adhesive bonding is a versatile fastening method for measurement equipment on impact-driven offshore monopile foundations. In an adhesive application campaign in 

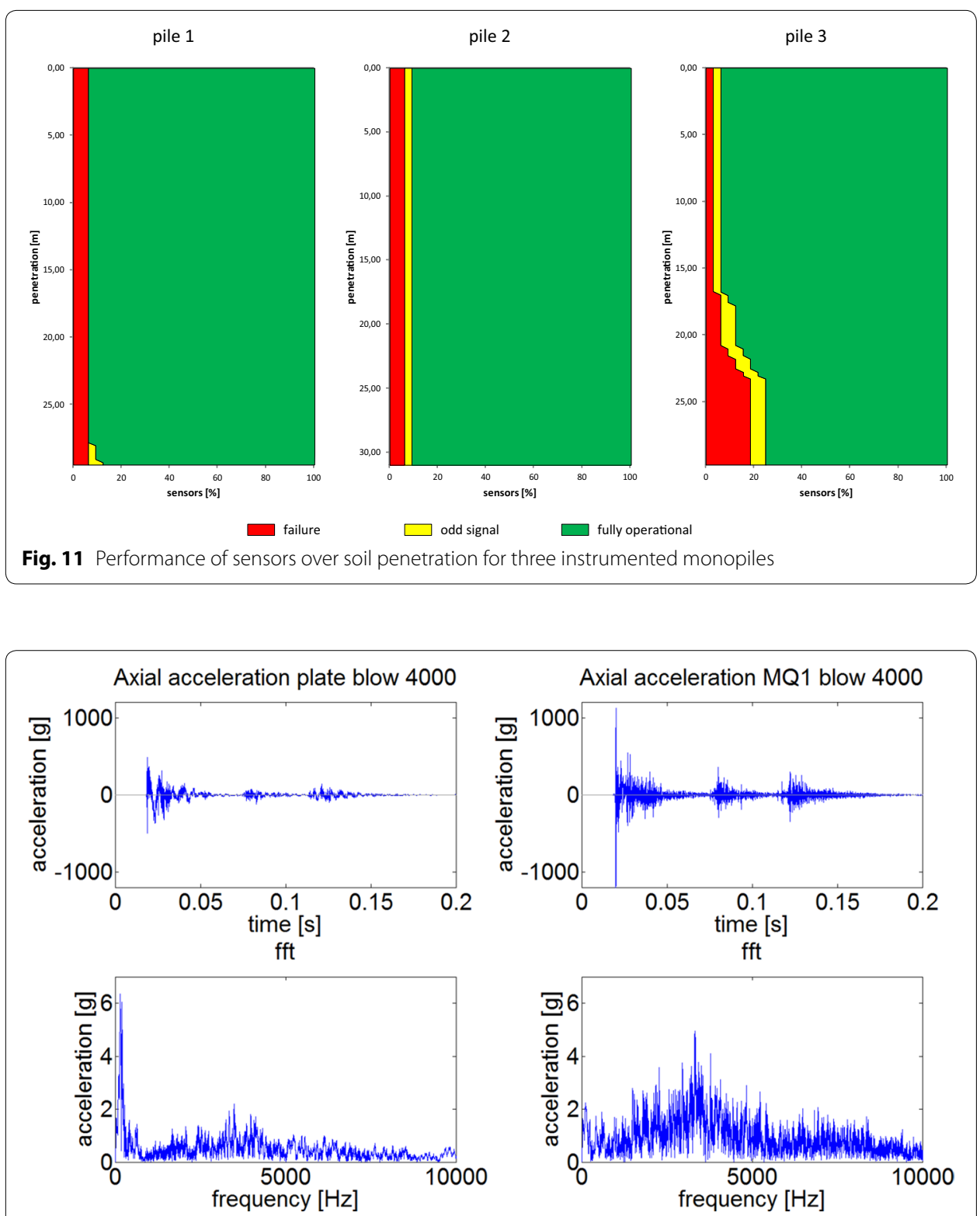

Fig. 12 Accelerations of mounting plate (left) and pile (right) in time (upper diagrams) and frequency (lower diagrams) domain

2014 three large monopile structures were successfully equipped and withstood the high energy pile driving into the North Sea. Looking at a specific adhesive technology, two cases have to be differentiated. On the one hand, small sensors for measuring the actual strain and accelerations of the large steel structure should be bonded with thin films and a structural strength, considering additional thin primer layers, if applicable. These layers assure minimal damping effects. On the other hand, all structures for protecting the sensors, cables, and monitoring equipment can be embedded into a thick elastic adhesive layer of about 5-10 mm and lower semi-structural strength. A boosted moisturecurable PUR adhesive applied on appropriate primer coatings was successfully applied with all hardware of the measurement system. 
The low quasi-static strength of less than $1 \mathrm{MPa}$ has to be considered and large bonding areas should be used. The impact tests on a compact specimen design to be mounted directly in a small Charpy test machine showed the well-known ability of elastic PUR adhesives for withstanding much higher impact loads than quasi-static loads. An approach on a similar specific energy application for a larger real mass bonded on a large area of the walls of a monopole, and a small specimen with low mass and small adhesive bond area was calculated. A repetitive application of only 39 blows with a specific configuration was performed and a small number of specimens achieved the target, others failed in different ways due to the not fully optimized method. In fact, not all specimens survived the full test period and showed that larger masses should be bonded on as much bonding area as possible and users of this technology should follow the joint design recommendations of best practices given in the literature. That leads in particular to the recommendation for bonding large structures (cable duct length of up to $50 \mathrm{~m}$ ) in separated smaller sections instead. This approach mainly avoids interfering vibrations with large mass and amplitudes. Finally, the elastic adhesive showed also an excellent sealing function, which made the use of extra sealing material on the sensor housings obsolete. Hence, adhesive bonding of the entire equipment for a fully autonomously pile driving monitoring of an impact driven large scale foundation structure for an offshore wind farm can be considered a completely new and very reliable application method.

\section{Authors' contributions}

GW coordinated all parts of the adhesive study, developed the adhesive concept, coordinated the preliminary testing and supervised the adhesive processes on the construction site. ES gave advice on materials, processes and interpretation of test results according to the challenging boundary conditions. KD gave advice on materials and testing concept and the interpretation of results. HS and PS planned and coordinated in equal parts all the work on the construction site, realized the data acquisition and assisted the pile driving campaign. They further contributed to the project with processing, statistics and interpretation of the data. CK implemented and performed the preliminary testing of adhesive joints (quasi-static and by impact). He tested and reported the presented solutions and assisted in the realization of all adhesive bonding on the construction site. JG supported the study in the preplanning phase and gave advice at all stages of the realization of the project to fit in the major construction process of the off-shore wind farm. All authors read and approved the final manuscript.

\section{Author details}

${ }^{1}$ Institute of Joining and Welding of Technische Universität Braunschweig IFS, Langer Kamp 8, 38106 Braunschweig, Germany. ${ }^{2}$ Institute for Soil Mechanics and Foundation Engineering of Technische Universität Braunschweig IGB, Beethovenstraße 51b, 38106 Braunschweig, Germany.

\section{Acknowledgements}

The project 'triad' (FKZ 0325681) was funded by the German Federal Ministry of Economics and Energy (see Fig. 13) on the basis of a decision of the German Bundestag.

\section{Supported by:}

Federal Ministry
for Economic Affairs
and Energy

on the basis of a decision by the German Bundestag

Fig. 13 Logo of funding organization BMWi 


\section{Competing interests}

The authors declare that they have no competing interests.

Received: 7 October 2015 Accepted: 7 November 2015

Published online: 24 November 2015

\section{References}

1. Stahlmann J, Kirsch F, Schallert M, Klingmüller O, Elmer K-H. Pfahltests—modern dynamisch und/oder konservativ statisch. In: Proceedings 4, Kolloquium 'Bauen in Boden und Fels', Technische Akademie Esslingen, 20-21 Jan 2004 ISBN 3-924813-55-8, 23-40; 2004.

2. Bruns B, Stein P, Kuhn C, Sychla H, Gattermann J. Hydro sound measurements during the installation of large diameter offshore piles using combinations of independent noise mitigation systems. In: Proceedings of 43rd international congress on noise control engineering, proceedings internoise 2014, 16-19 November 2014, Melbourne: 2014.

3. Standard ISO 179-1. Plastics — determination of Charpy impact properties - part 1: non-instrumented impact test (ISO 179-1:2010). Geneva, Switzerland: International Organization for Standardization (ISO); 2010.

4. Standard ISO 11343:2003. Adhesives-determination of dynamic resistance to cleavage of high-strength adhesive bonds under impact conditions - Wedge impact method. Geneva, Switzerland: International Organization for Standardization (ISO); reviewed 2013.

5. Adams RD. The pendulum impact test for adhesives and adhesive joints. In: da Silva LFM, Dillard DA, Blackman B, Adams RD, editors. Testing adhesive joints—best practices. Weinheim: Wiley-VCH; 2012. p. 280-3.

6. Hunke R, Bieker C, Klapp O, Schlimmer M. Dickschichtklebeverbindungen in Strukturen von Transportfahrzeugen, Proceedings 14. Symposium Verbundwerkstoffe und Werkstoffverbunde, Wien, Austria, 2-4 July 2003. Weinheim: Wiley-VCH; 2003, p. 907-12.

7. Großkurth L, Schlimmer M. Fracture mechanical tests of thick film bonded joints German Title: Bruchmechanische Untersuchungen von Dickschichtklebungen, Proceedings Werkstoffprüfung, 23. Vortrags- und Diskussionstagung, Berlin; 2005, p. 377-82.

8. Moulds RJ. Design and stress calculations for bonded joints. In: Cognard P, editor. Handbook of adhesives and sealants, vol. 2. Oxford: Elsevier Ltd; 2006. p. 197-231.

9. Pröbster M. Elastisch Kleben. Wiesbaden: Springer Vieweg Verlag; 2013

\section{Submit your manuscript to a SpringerOpen ${ }^{\circ}$ journal and benefit from:}

- Convenient online submission

- Rigorous peer review

- Immediate publication on acceptance

- Open access: articles freely available online

- High visibility within the field

- Retaining the copyright to your article

Submit your next manuscript at $>$ springeropen.com 